\title{
Hadronization of quarks and correlated di-hadron production in hard scattering
}

\author{
Harut Avakian * \\ Jefferson Lab \\ E-mail: avakianejlab.org
}

\begin{abstract}
Understanding the dynamics of partons in general, and non perturbative sea, in particular, will be crucial for the understanding of strong interactions. Correlations of the spin of the target or/and the momentum and the spin of quarks, combined with final state interactions define the azimuthal distributions of produced particles. Production of correlated hadron pairs, in addition, will play an increasingly important role in the interpretation of pion electroproduction data in general, and hadronization process of quarks, in particular. More significant, than originaly anticipated, fraction of pions coming from correlated di-hadrons, indicated by recent CLAS12 measurements, and supported by various realistic models describing the hadronization process, may have a significant impact on various aspects of data analysis, including the modeling, composition, and interpretation of semi-inclusive DIS data, as well as calculations of radiative corrections. Studies of the nucleon structure beyond the traditional leading twist and current fragmentation of inclusive hadrons, provide qualitatively new tools to study the nucleon structure and extension of large $x$ region well covered by JLab, to large $Q^{2}$ at the EIC where it will be very important in interpretation of the data from JLab.
\end{abstract}

XXVII International Workshop on Deep Inelastic Scattering and Related Subjects (DIS2019) -

8-12 April, 2019

Torino, Italy

*for the CLAS Collaboration 


\section{Dihadron production}

Semi-inclusive deep inelastic scattering (SIDIS) has emerged as a powerful tool to probe nucleon structure through measurements of spin azimuthal asymmetries, providing direct access to Transverse Momentum Distributions of partons (TMDs). TMDs have been widely recognized as one of key objectives of the JLab $12 \mathrm{GeV}$ upgrade, and can be relevant for studies in proton colliders, even at the LHC energies. In the last years, there has been flourishing activity on TMDs, thanks to pioneering experimental measurements and novel theoretical ideas. First measurements of spin and azimuthal asymmetries in various processes were already accomplished at JLab and many more are approved to take part in both near and long term future. Correlations of the spin of the target or/and the momentum and the spin of quarks, combined with final state interactions define the azimuthal distributions of produced particles. Understanding the quark and gluon structure of hadrons within the theoretical framework of quantum chromodynamics (QCD), requires detailed understanding of correlated di-hadron production in hard scattering processes. Measurements of spin-azimuthal asymmetries (SSAs) in di-hadron production indicate that final state hadrons tend to be correlated.

The invariant mass distributions of di-hadrons from different SIDIS and e+e- experiments indicate, the fraction of pions from vector meson (VM) decays may be very significant. While, single hadron production, being part of correlated di-hadron production, in principle could be described in the factorised approach, as a convolution of set of different spin-dependent and independent distribution and fragmentation functions [1], there are certain applications where the simple fragmentation functions, depending on the fraction of the virtual photon energy carried by the hadron, $z$, and even transverse momentum generated in the fragmentation $p_{T}$, may not be enough for precision description of hard processes. The list of these applications, some of them critical for interpretation of the data, includes modeling of hadronization of polarized quarks, procedures for accessing transverse momentum of quarks, fraction of secondary lepton pairs produced in electroproduction, and even the procedure for radiative corrections in SIDIS. The polarized fragmentation functions, in particular, can be different, sometimes with opposite signs, depending on whether the hadron is produced directly or through intermediate correlated dihadron, primarily a vector meson [2, 3, 4]. A simple example discussed by Czyzewski [3], using Artru model for transverse quark fragmentation, is shown in Fig.1. To hadronize into a spin-1 particle from orbiting $q \bar{q}$ in a given direction, opposite spin orientation of fragmenting quark will be preferable, compared to spin- 0 . In addition, in case the fragmentation of $u$-quarks goes predominantly through $\rho^{0}$ and $\rho^{+}$production, compared to spin- 0 production, the relative fraction of $u \bar{u}$ and $d \bar{d}$ may be relevant. Measurements of flavor asymmetries in sea quark distributions performed in DY experiments, indeed indicate very significant non-perturbative effects at large Bjorken- $x$, where the valence quarks are relevant [5]. Older measurements by NMC [6] indicated that integrated $\bar{d}$ is more than integrated $\bar{u}$. The measurements by E866 collaboration [7], and more recently by SeaQuest [8] suggest that $\bar{d}$ is significantly larger than $\bar{u}$ in the full accessible $x$-range. That may have an observable effect on the relative fraction of $\rho^{+}$and $\rho^{0}$ production in the hadronization process. The non-perturbative $q \bar{q}$ pairs, are also correlated with spins, and play a crucial role in spin orbit correlations in general, and single-spin asymmetries measured by various experiments in last few decades, in particular.

The full event Monte-Carlo generators (MCs) [9, 10, 11] based on LUND fragmentation 


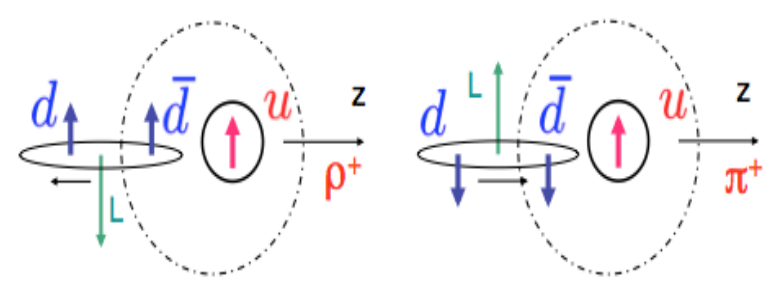

Figure 1: Production of spin-1 (left) and spin-0 (right) particles will pick $q \bar{q}$ pairs orbiting in opposite directions.

model, with default settings using $50 \%$ for fraction of spin- 1 versus spin- 0 hadrons produced by quarks, which were used so far by SIDIS experiments at different energies, suggest that the dominating fraction of pions are indeed coming from vector meson decays (see Fig.2). The effects of the production and strong decays of vector mesons during quark hadronization have been studied also in NJL model [12], indicating that products of the vector mesons play predominant role in the contributions to fragmentation functions for pseudoscalar hadrons. Recent measurements of dihdrons produced in $e^{+} e^{-}$collisions from Belle [13] also support the significant role VMs play in the overall sample of inclusive pions.

Measurements of beam SSA, $A_{L U}$ sensitive to quark-gluon-quark correlations [14, 15, 16, 17], performed by CLAS Collaboration at Jefferson Lab, indicate that there is a significant asymmetry in the single-pions sample originating from rho-mesons, which is also very different for different vector mesons. The size of the asymmetry reaches $\sim 20 \%$ (see Fig. 2 ), and is opposite for $\pi^{+}$originating from exclusive $\rho^{+}$and $\rho^{0}$ decays [18]. In addition since the spin dependent fragmentation (Collins function) of rho mesons was predicted to have an opposite sign [3], the final interpretation of pion asymmetries will be very sensitive to relative fraction of pions coming from vector meson decays. CLAS preliminary measurements [19] indicated a very significant non-zero beamspin asymmetry $A_{L U}$ both on ${ }^{2} \mathrm{H}$ and $\mathrm{NH}_{3}$ targets. Interpretation of kinematical dependences of different SSAs, in general, both leading and higher twist, measured in SIDIS experiments in past 20 years [20] may have significant dependence on the kinematical distributions of the fraction of pions coming from exclusive and semi-inclusive rhos.

The comparison of di-hadron multiplicities measured by CLAS12 and simulation, based on the Lund fragmentation [10] and full GEANT4 simulation of the CLAS12 detector, is shown on Fig.3. The good agreement, indicates, that the Lund string fragmentation is very realistic, and works perfectly at low energies, where the multiplicities of hadrons are pretty low, and even in the exclusive limit it is consistent with data. Some explanation of that, at first glance unexpected finding, can be obtained from comparing the cross sections of exclusive final states, measured at JLab. Comparison of exclusive $\pi^{0}$ [21] versus exclusive $\rho^{0}$ [22] production on proton and exclusive $\pi^{+}$[23] versus exclusive $\rho^{+}$[24] on proton indicates that rhos in general have higher probability to be produced than corresponding charged pions with similar quark structure (see Fig. 4). Higher cross-sections for exclusive $\rho^{+}$than for $\rho^{0}$ suggests, that gluon exchange diagrams, expected to dominate at high energies, are suppressed, and vector mesons are produced mainly in hard scattering over quarks, similar to case of SIDIS.

The possible fraction of pions coming from diffractive $\rho^{0}$ decays has been studied by all 

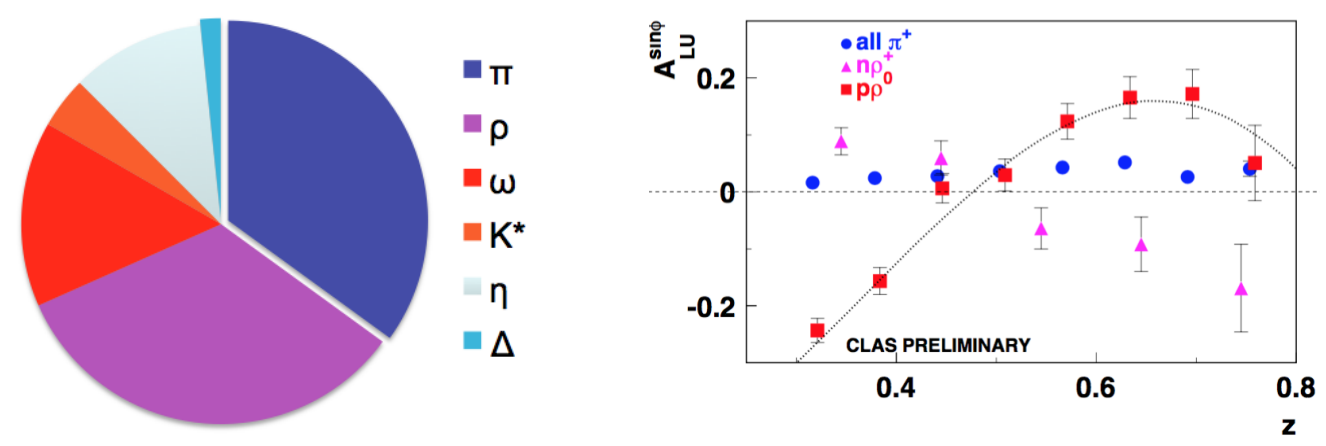

Figure 2: Left panel: The fraction of pions in $e p \rightarrow e^{\prime} \pi X$ in HERMES kinematics (27.5 GeV), produced directly from the string in PYTHIA MC (shown as $\pi$ ) and from different decay channels $\left(\rho, \omega, K^{*}, \eta\right.$ and $\Delta$ ). Right panel: The $A_{L U}^{\sin \phi}$ asymmetry measured by CLAS on hydrogen target, for final state $\pi^{+}$originating from exclusive $\pi^{+} \pi^{-}$events dominated by $\rho^{0}$ (red squares), from exclusive $\pi^{+} \pi^{0}$ events dominated by $\rho^{+}$ (magenta triangles) and integrated over all sources (blue circles) [18].

collaborations involved in SIDIS studies and some corrections due to this fraction have been applied $[25,26,27,28]$ in the past. The fraction of pions coming from diffractive rho decays may indeed be a concern, as those pions may not be easily related to quark distribution functions, which are the main focus of SIDIS studies. The rhos generated from quarks in the process of hadronization, were always considered as a part of the regular fragmentation function of quarks into hadrons. The pions coming from decays will, nevertheless, have some peculiar features which can be studied from SIDIS data, and also investigated in details in MC.
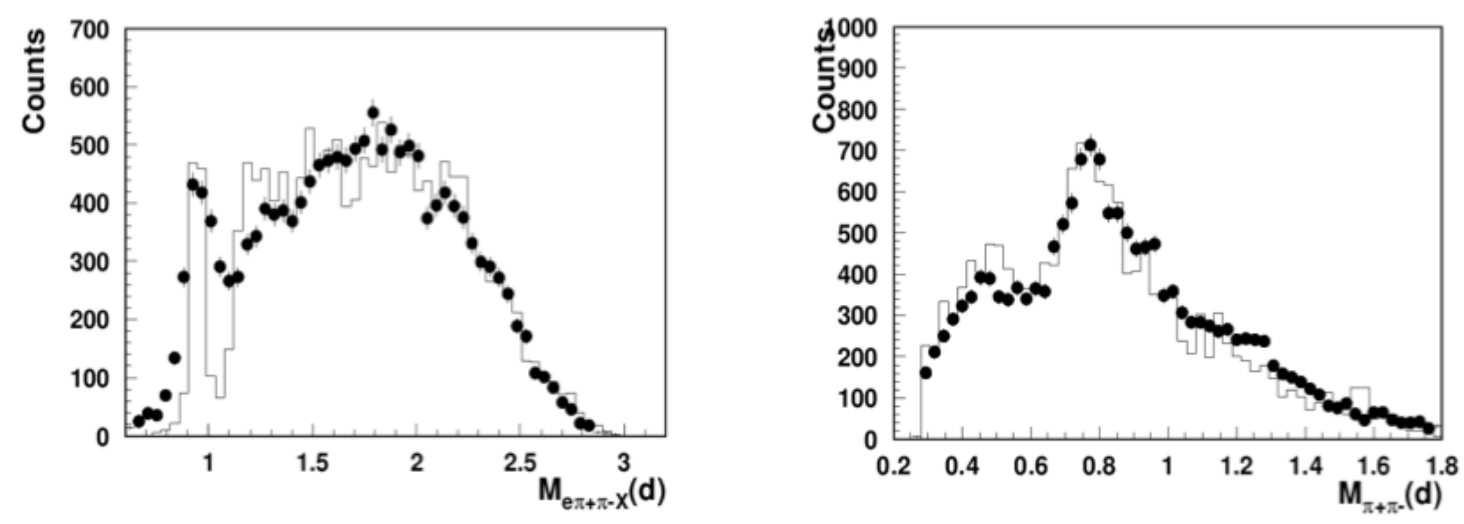

Figure 3: The comparison of missing mass (left) and invariant mass of reconstructed hadron pairs measured by CLAS12 at $10.6 \mathrm{GeV}$ with deuteron target with events generated using LUND MC, and passing the full GEANT simulation and clas 12 reconstruction. Both event samples represent the raw response, and are normalized by the total number of inclusive electrons.

Good agreement of data and Lund MC, allows some more detailed studies of fraction of pions coming from correlated dihadrons in general, and specific vector mesons, in particular. The $P_{T^{-}}$ distributions of fragmenting neutral rhos, and corresponding decay $\pi^{ \pm}$, are shown in Fig.5. In average the decay pions occupy lower $P_{T}$ range. The ratio of pions coming directly from string fragmentation to ones coming from rho decays, indicates that fraction of pions from rho decays is 

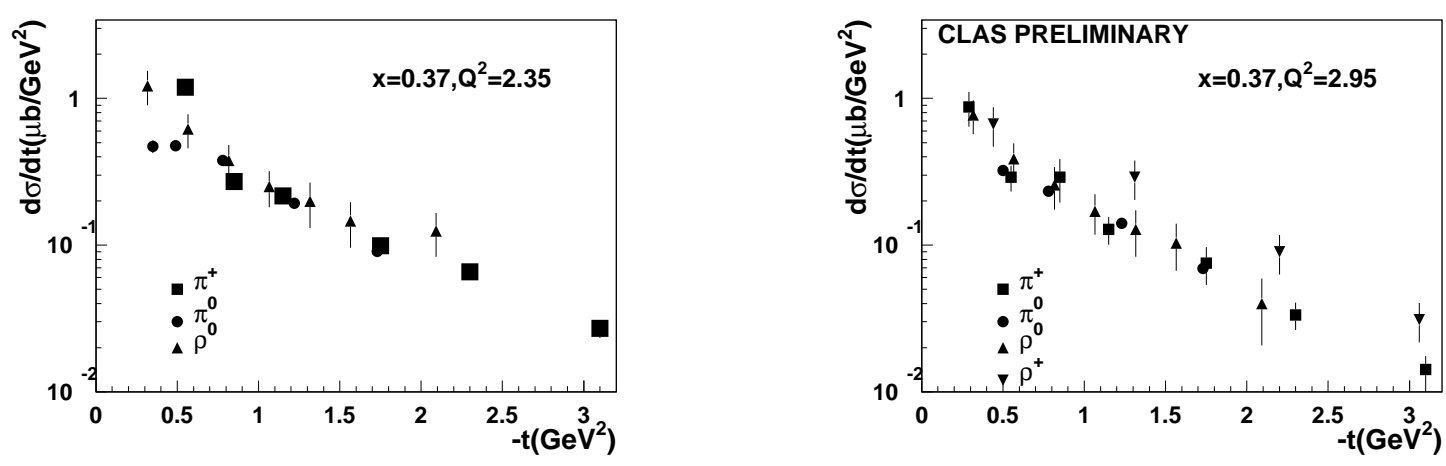

Figure 4: Dependence on momentum transfer $t$ for cross sections of exclusive production of $\pi^{0}, \pi^{+}, \rho^{0}, \rho^{+}[21,23,22,24]$ on proton target from CLAS measurements at Jefferson Lab at $6 \mathrm{GeV}$.

most significant, most likely dominating at small $P_{T}$ (see Fig.5). At large $P_{T}$ the fraction of direct pions is increasing, for all $z$-bins.
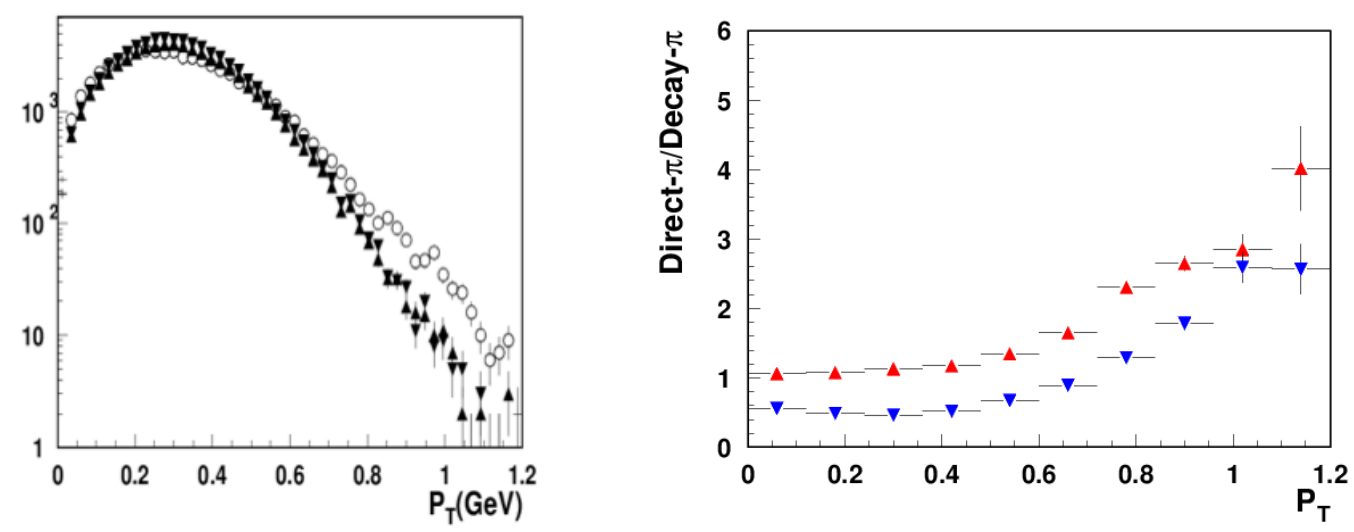

Figure 5: The $P_{T}$-dependence of the $\rho^{0}$ (open circles) and decay pions (triangles) in CLAS12 kinematics at $10.6 \mathrm{GeV}$. The right panel shows the ratio of the fractions of $\pi^{+}$(triangle sup) and $\pi^{-}$(triangles down) produced directly from string, and from decays, as a function of $P_{T}$.

The overall fraction of pions coming from VMs could be tuned through some model parameters in PYTHIA [11]. While the absolute numbers may change depending on actual fraction of correlated dihadrons in the hadronization process, the qualitative dependence on kinematical variables will be similar, supported by good agreement of data and MC. The overall fraction may be different in reality, and could be studied comparing the kinematical dependencies of di-hadrons and 
single hadrons. Higher fraction of VMs produced in the hadronization process will change also the number of lepton pairs produced in the hadronization process, which may have some observable impact on Drell-Yan measurements. Even though the branching ratio of $\rho^{0}$ and $\omega$ decays to lepton pairs is fairly low (4.72E-5 and 7.4E-5, respectively), they are produced with relatively high energies, and may show up in combinatorial background at large invariant masses. Significant fraction of $e^{\prime} e^{+} e^{-} X$ events measured by CLAS12 detector provide important info on the absolute number of VMs produced in DIS, allowing for better understanding of the details of hadroniztion process needed also for a realistic tune of LUND MCs.

Comparison of MC simulations with increased fractions of VMs in the overall sample of pions, indeed indicate that fraction of VMs tend to increase in the set of relatively smaller $z$ and $P_{T}$ of pions at expense of larger $z$ ones, improving the overall agreement. Another important implication of larger fraction of pions coming from VM decays will be the increase of the average transverse momentum of quarks, needed for better description of data, allowing to describe large transverse momentum of pions, where direct pions dominate (see Fig. 5). That may help, in particular, in description of the large $P_{T}$ region of SIDIS, where simple Gaussians, typically derived from the relatively low $P_{T}$ range $(\sim 0.2-0.8 \mathrm{GeV})$, fail to describe the data [28]. Extracted average widths of partonic transverse momentum distributions will, thus, change with the range of involved transverse momentum of hadrons.

Interestingly enough, picture reverses for the dependence on $P_{T} / z$ and $P_{T} / z / Q$, which are relevant for accessing the transverse momentum of quarks, and for definition of the kinematical region, where the perturbative calculations are safe to apply, assuming $P_{T} / z / Q>1$. The Fig.6 shows the dependence on $P_{T} / z / Q$ of the fraction of pions from rho decays. The direct pions, dominating the large $P_{T}$ appear at smaller $P_{T} / z$, due to in average larger $z$, while large $P_{T} / z$ are populated by smaller $z$ pions, which in fact come from large $z$ VMs, produced in the non-perturbative region $P_{T} / z / Q<1$ (see Fig.6). Understanding the production mechanism, may change the way factorization is applied. The right panel of Fig.6 shows $P_{T} / z / Q$ distributions calculated using the $z$ of the pion (triangles), and the $z$ of the parent $\rho^{+}$(circles). The transverse momentum of the original quark will certainly have more connection to the $z$ and $P_{T}$ of parent $\rho$, produced in PYTHIA directly from the string, than to the decay pion with much lower $z$. Indeed, one of the main reasons of large discrepancies observed in comparison with COMPASS data in Ref. [29] may be the application of perturbative calculations in the region of $P_{T} / z / Q>1$ with significant fraction of pions coming from decays of vector mesons produced in non-perturbative region with $P_{T} / z / Q<1$.

Another application, where the actual source of pions matter, are the radiative corrections (RC) to measurements, which are indispensable part of experimental measurements in leptoproduction, when the incoming and scattered leptons radiate photons (see Fig. 7) which change the kinematics of the hard scattering, requiring unfolding of original cross section from measurements integrated over the whole accessible $W$-range. The actual $W$-range contributing to a single kinematical point in $Q^{2}, W^{2}$ estimated using the RADGEN code [30] is shown in Fig. 7. Due to radiation of photons, extending in energy up to the energy of original beam, the final measurements, get contributions from a wide spectra of kinematics outside of intended measurement, including exclusive processes. In case the hadronization goes predominantly through production of correlated dihadrons, the self consistent approach for accounting the radiative corrections, should include the structure functions describing their production, including the exclusive limit. 

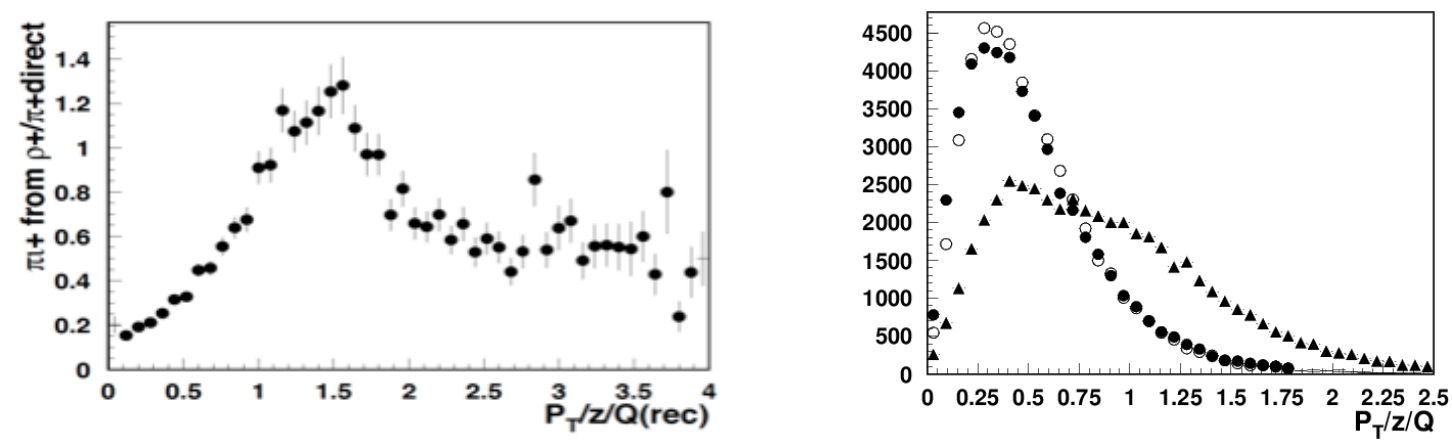

Figure 6: The $P_{T} / z / Q$-dependence of pions in CLAS12 kinematics at $10.6 \mathrm{GeV}$. The left panel shows the ratio of fractions of inclusive $\pi^{+}$from $\rho^{+}$decays as a function of $P_{T} / z / Q$. The right panel shows the distribution over $P_{T} / z / Q$ for $\pi^{+}$calculated using the $z$ of the pion (triangles) and $z$ of the parent $\rho^{+}$(filled circles). The open circles show the same distribution calculated using the $P_{T}$ and $z$ of the parent $\rho^{+}$.
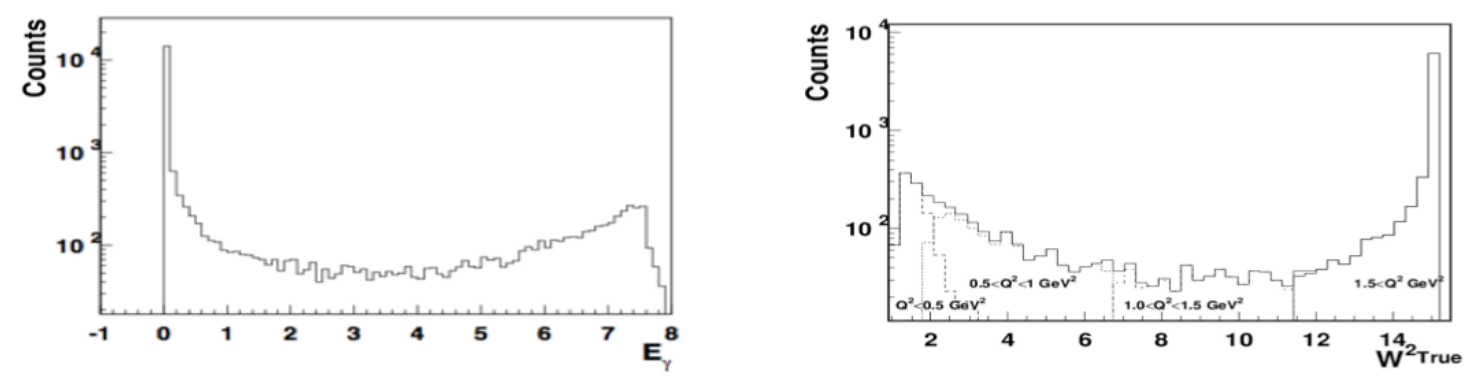

Figure 7: Left panel: The spectra of radiated photons from incoming electron beam of $10.6 \mathrm{GeV}$ electrons at JLab for a given kinematical point with $x, y, Q^{2}, W^{2}\left(0.12,0.8,1.9 \mathrm{GeV}^{2}, 15.1 \mathrm{GeV}^{2}\right)$. Right panel: The actual $W$-range contributing to experimental point with $Q^{2}=1.9$ and $W^{2}=15.1 \mathrm{GeV}^{2}$, introducing a 1.24 factor for radiative correction to the DIS cross section.

The understanding of the contributions to the final transverse-momentum dependence of different azimuthal moments in the cross section will require detailed studies of different contributions. Monte-Carlo (MC) event generators accounting for spin-orbit correlations in di-hadron production will be crucial in the study of the sensitivity of final single and di-hadron observables to various experimental uncertainties including acceptances, resolutions, phase space limitation, and radiative corrections in SIDIS for different model inputs of underlying structure functions. There were several attempts to add some spin-orbital contributions to PYTHIA [31, 32]. Adding the spin-orbit correlations in a self consistent way to account for relevant correlations, however, it appeared to be a complicated task, requiring very significant combined theory and computing efforts. Additional structure functions related to those correlations will be critical for consistent account of RC in relevant measurements of different azimuthal modulations in single and di-hadron cross-sections, and related SSAs [33]. To account for all those correlations, one needs a comprehensive MC generator, which can introduce correlations in a self consistent way. A prototype of such a generator (TMD-Gen), has been developed by S. Gliske [34] in collaboration with Pavia group. While the development of that type of dedicated event generators, will also require a significant effort from the TMD community, it will certainly require significantly less manpower than upgrade of the full 
event generators like PYTHIA or HERWIG, to account for spin-orbit and quark-gluon-quark correlations, relevant in the region of relatively large $x$, where non-perturbative effects in general, and spin-orbit correlations, in particular, are significant.

In summary, the CLAS12 data support predictions from different MCs of very significant fraction of inclusive pions coming from correlated dihadrons. The observables for pions from decays of vector mesons have peculiar spin and momentum dependences and may require different RC, modeling, and interpretation of observables sensitive to transverse momentum of quarks. The interpretation of di-hadron production in SIDIS, as well as interpretation of single-hadron production, are intimately related to contributions to those samples from correlated semi-inclusive and exclusive di-hadrons in general, and rho mesons, in particular.

We thank A. Kotzinian, A. Bacchetta, and R. Seidl for their help and discussions. This work was supported by the U.S. Department of Energy under Contract No. DE-AC05-06OR23177.

\section{References}

[1] J. Collins, Foundations of perturbative QCD (Cambridge University Press, 2013).

[2] X. Artru, J. Czyzewski and H. Yabuki, Z.Phys. C73 (1997) 527, arXiv: hep-ph/ 9508239.

[3] J. Czyzewski, Acta Phys. Polon. 27 (1996) 1759, arXiv: hep-ph/ 9606390.

[4] X. Artru and Z. Belghobsi, AIP Conf.Proc. 1444 (2011) 97.

[5] M. Alberg and G.A. Miller, arXiv:1712.05814 (2017), arXiv: 1712.05814.

[6] New Muon, P. Amaudruz et al., Phys. Rev. Lett. 66 (1991) 2712.

[7] G.T. Garvey and J.C. Peng, Prog. Part. Nucl. Phys. 47 (2001) 203, arXiv: nucl-ex/ 0109010.

[8] K. Nagai, JPS Conf. Proc. 13 (2017) 020051.

[9] G. Ingelman, A. Edin and J. Rathsman, Comput.Phys.Commun. 101 (1997) 108, arXiv:hep-ph/9605286.

[10] L. Mankiewicz, A. Schafer and M. Veltri, Comput. Phys. Commun. 71 (1992) 305.

[11] T. Sjostrand, S. Mrenna and P.Z. Skands, JHEP 0605 (2006) 026, arXiv : hep-ph / 0603175.

[12] H.H. Matevosyan, A.W. Thomas and W. Bentz, Phys.Rev. D88 (2013) 094022, arXiv:1310.1917.

[13] Belle, R. Seidl et al., Phys. Rev. D96 (2017) 032005, arXiv: 1706.08348.

[14] A.V. Efremov, K. Goeke and P. Schweitzer, Nucl. Phys. A711 (2002) 84, hep-ph/ 0206267.

[15] M. Burkardt, Phys. Rev. D88 (2013) 114502, arXiv: 0810.3589.

[16] B. Pasquini and S. Rodini, arXiv:1806.10932 (2018), 1806.10932.

[17] S. Bastami et al., JHEP 06 (2019) 007, arXiv: 1807.10606.

[18] CLAS, H. Avakian and L. Elouadrhiri, Gerasimov-Drell-Hearn sum rule and its extensions. Proceedings, 3rd International Symposium, GDH 2004, Norfolk, USA, June 2-5, 2004, pp. 228-232, 2004.

[19] CLAS, S. Pisano, EPJ Web Conf. 73 (2014) 02008. 
[20] H. Avakian, B. Parsamyan and A. Prokudin, Riv. Nuovo Cimento 42 (2019) 1.

[21] CLAS, I. Bedlinskiy et al., Phys. Rev. C90 (2014) 025205, arXiv: 1405 . 0988 , [Addendum: Phys. Rev.C90,no.3,039901(2014)].

[22] CLAS, S.A. Morrow et al., Eur. Phys. J. A39 (2009) 5, arXiv: hep-ex/0807. 3834.

[23] CLAS Collaboration, K. Park et al., (2012), arXiv:1206.2326.

[24] A. Fradi, AIP Conf. Proc. 1374 (2011) 537, arXiv:1010.1198.

[25] CLAS Collaboration, H. Avakian et al., Phys. Rev. D69 (2004) 112004, hep-ex/ 0301005.

[26] HERMES, A. Airapetian et al., Phys. Lett. B648 (2007) 164, hep-ex/ 0612059.

[27] CLAS Collaboration, H. Avakian et al., Phys. Rev. Lett. 105 (2010) 262002, arXiv:hep-ex/1003.4549.

[28] COMPASS, M. Aghasyan et al., Phys. Rev. D97 (2018) 032006, arXiv: 1709.07374.

[29] J.O. Gonzalez-Hernandez et al., Phys. Rev. D98 (2018) 114005, arXiv: 1808.04396.

[30] I. Akushevich, H. Bottcher and D. Ryckbosch, Monte Carlo generators for HERA physics. Proceedings, Workshop, Hamburg, Germany, 1998-1999, pp. 554-565, 1998, hep-ph/9906408.

[31] H.H. Matevosyan, A. Kotzinian and A.W. Thomas, Phys. Rev. D95 (2017) 014021, arXiv:1610.05624.

[32] A. Kerbizi et al., Phys. Rev. D97 (2018) 074010, arXiv:1802.00962.

[33] I. Akushevich and A. Ilyichev, (2019), arXiv:1905.09232.

[34] S. Gliske, A. Bacchetta and M. Radici, Phys. Rev. D90 (2014) 114027, arXiv: 1408.5721 , [Erratum: Phys. Rev.D91,no.1,019902(2015)]. 